\title{
Incidencia de la actividad minera en el desarrollo local sostenible: el caso del Cantón Camilo Ponce Enríquez
}

\author{
Incidence of mining activity on sustainable local development in the case of \\ the canton camilo ponce enríquez
}

Guillermo Esteban Matute Saquicela. ${ }^{1}$, Daniele Covri Rivera. ${ }^{2} \&$ Yonimiler Castillo Ortega. ${ }^{3}$

Recibido: 21-12-2020 / Revisado: 19-12-2020 /Aceptado: 14-01-2021/ Publicado: 05-01-2021

\begin{abstract}
.
DOI: $\underline{\text { https://doi.org/10.33262/concienciadigital.v4i1.1.1555 }}$

Introduction, The impacts of mining are evaluated with different sets of indicators and targets at the global level. This paper presents a review of the literature associated with the mining activity from which a survey has been prepared that was applied to the actors of the mining sector of the Camilo Ponce Enríquez Canton. Objective is to analyze sustainable local development and the incidence of mining activity, identifying the essential relationships between mining activity and economic, social, territorial and environmental variables in the case of the Camilo Ponce Enríquez canton. Methodology. It should be noted that this study presents a mixed approach, since it uses more than one method for its execution, therefore, this was a cross-sectional study which allowed the identification of the essential relationships between mining activity and the different variables Results. After conducting an analysis of the entire population of the Camilo Ponce Enríquez song, a scenario of 72 people whose income depends directly or indirectly on mining was involved, dealing with the topics Economy, security, Employment, Education, Land use, territorial aspects, Demography, Environment, Human Rights. Conclusion, it must change its approach and be oriented mainly

${ }^{1}$ Universidad Católica de Cuenca, Posgrado, Maestría en Desarrollo Local Mención en Ordenamiento Territorial, Cuenca, Ecuador, guimomatutesaquicela@ hotmail.com, https://orcid.org/0000-0002-7710-5199

${ }^{2}$ Universidad Católica de Cuenca, Posgrado, Maestría en Desarrollo Local Mención en Ordenamiento Territorial, Cuenca, Ecuador, daniel.covri@ucacue.edu.ec, https://orcid.org/0000-0002-2495-0399

3 Universidad Católica de Cuenca, Posgrado, Maestría en Desarrollo Local Mención en Ordenamiento Territorial, Cuenca, Ecuador, ycastillo@ucacue.edu.ec, https://orcid.org/0000-0002-7710-5199
\end{abstract}


to the well-being of the citizens of the area, it must promote an economy of "circular return" and with a solidarity approach. Differentiating them from the soils that will be used as green spaces, for agricultural production, recreation, and from the areas suitable for inhabiting.

Keyboards: Mining, social impact, employment, health, human rights

\section{Resumen.}

Introducción, Los impactos de la minería se evalúan con diferentes conjuntos de indicadores y metas a nivel global. Este trabajo presenta una revisión de la literatura asociada a la actividad minera a partir de la que se ha elaborado una encuesta que se aplicó a los actores del sector minero del Cantón Camilo Ponce Enríquez. Objetivo es analizar el desarrollo local sostenible y la incidencia de la actividad minera, Identificando las relaciones esenciales entre la actividad minera y las variables económicas, sociales, territoriales y ambientales en el caso del cantón Camilo Ponce Enríquez. Metodología. Cabe señalar que este estudio presenta un enfoque mixto, ya que emplea más de un método para su ejecución, así pues, éste fue un estudio transversal lo que permitió Identificar las relaciones esenciales entre la actividad minera y las diferentes variables Resultados. Después de realizar un análisis a toda la población del canto camilo Ponce Enríquez se involucró a un escenario de 72 personas cuyos ingresos dependen directa o indirectamente de la minería tratando los temas Economía, seguridad Empleo, Educación, Uso del suelo, aspectos territoriales, Demografía, Medio Ambiente, Derechos humanos. Conclusión, debe cambiar su enfoque y orientarse principalmente al bienestar de los ciudadanos de la zona, deben promover una economía de "retorno circular" y con enfoque solidario. Diferenciándolos de los suelos que se utilizarán como espacios verdes, para producción agrícola, recreativos, y de las zonas aptas para habitar.

Palabras claves: Minería, impacto social, empleo, salud, derechos humanos

\section{Introducción.}

Los minerales, recursos importantes para el sector industrial de los países del mundo y esenciales para su bienestar y desarrollo económico, son uno de los pilares sobre los que se sostienen las sociedades modernas. Por lo dicho, resulta importante investigar entre otras preocupaciones, la incidencia social que tiene la minería en las regiones en las que se este sector productivo realiza sus actividades, el presente estudio pretende dar respuesta a las siguientes preguntas de investigación: 
Q1: ¿Cuáles son los principales problemas sociales que se reportan en investigaciones realizadas a nivel global sobre las actividades mineras y el impacto percibido por cada uno de estos problemas?

Q2: ¿Cuál es la percepción de las personas del Cantón Ponce Enríquez en cuando a los factores reportados como más preocupantes en la encuesta aplicada?

Q3: ¿Cuál es el papel del gobierno seccional del Cantón para conseguir un desarrollo adecuado, sostenible y sustentable de las actividades mineras?

Muchos de los Objetivos de Desarrollo Sostenible (ODS) establecidos por las Naciones Unidas, conocidos también como Objetivos Mundiales (adoptados por los Estados Miembros de la ONU en 2015 como un llamado para poner fin a la pobreza, proteger el planeta y garantizar que todas las personas gocen de prosperidad y paz para el 2030 (Rosa, 2017)), no podrían alcanzarse sin contar con insumos tales como los metales y los minerales, mismos que son importantes materias primas para el sector manufacturero y cuya explotación y obtención crea empleo y valor agregado a lo largo de los procesos que terminan en su suministro. Por otra parte, la producción de estas importantes y valiosas materias primas minerales puede generar impactos negativos en aspectos como el social y el ambiental, limitando de ese modo en las zonas donde se realizan las actividades mineras, la consecución de otros objetivos importantes para el anhelado desarrollo sostenible (por ejemplo: agua limpia, buena salud, acción climática).

Por lo dicho, es obvia la actual preocupación existente en varios territorios del plantea por la escases de ciertos recursos minerales, escasez causada por el consumo masivo de estos recursos, factor vinculado al aumento exponencial del número de personas que habitan el planeta, ha hecho más notable la relevancia política de estas materias primas desde hace varias décadas (Dewulf et al., 2016).

En el caso de Latinoamérica el desarrollo de las actividades mineras sigue siendo necesaria ya sea para el desarrollo de la sociedad o como importante aporte económico de los países de la región. Cabe destacar la no coherencia de las políticas públicas para el mejoramiento del manejo socioambiental de las actividades mineras (Ríos, 2018).

Para (Saade Hazin, 2013), a pesar de que la minería se presenta como una de las actividades que atrae más inversión extranjera para los países de América Latina y el Caribe (ALC), sigue siendo también una de las actividades que produce mayores impactos negativos sobre el ambiente, ya que en comparación con el promedio de otros sectores económicos, genera 5 veces más emisiones de gas efecto invernadero por dólar de producción. En este contexto, los costes socio ambientales que acarrea la actividad minera, pueden ser grandes so se consideran los impactos que tiene sobre el agua, el aire, el suelo, en fin en el ecosistema donde se desarrollan actividades mineras, problemas cuya solución podría ocasionar mayor costo (Twerefou, 2009). 
En este orden de ideas, en Ecuador el impacto que causan las actividades mineras y las políticas que rigen el accionar y funcionamiento de las empresas que realizan dichas actividades en el país no son diferentes a los reportados en los países de ALC, resultando importante señalar en este punto que esta actividad en nuestro país, tiene un precedente histórico ya que se han encontrado minas con potencial industrial que fueron explotadas desde la presencia de los españoles a suelo patrio (1492), este hecho se presenta como un hito en la historia minera de nuestro país y de la región.

Políticas de estado dictaminan que sea el Instituto Nacional de Investigación GeológicoMinero-Metalúrgico (Inigemm), el organismo encargado de sistematizar, generar, administrar y focalizar la información geológica en nuestro país, y tiene como uno de sus objetivos la promoción del desarrollo sustentable y sostenible de los recursos minerales del territorio y la prevención de amenazas geológicas y las que ocasionase el ser humano (Toapanta, 2017).

Este trabajo tiene como objetivo analizar los principales problemas sociales reportados en la literatura analizada y evaluar su impacto según la percepción de los actores de la industria minera de Cantón Camilo Ponce Enríquez, Azuay - Ecuador.

\section{Marco Teórico}

La revisión de literatura especializada sobre los problemas sociales que genera la minería en otros lugares del planeta se empleó para determinar los impactos sociales de mayor ocurrencia en los sectores donde se realizan actividades mineras, el estudio empleó artículos y documentos validados que fueron buscados en bases de datos académicas como es el caso de Google Scholar, Springer, Scopus, donde se emplearon las palabras clave: minería, impacto social, empleo, salud, derechos humanos sumándose a estos los términos: economía, ambiente, seguridad, uso de suelo y migración, además se verificó que los trabajos revisados cumplan el criterio de ser estudios académicos internacionales que hayan sido publicados entre 2010 y 2017, se consideró este período de tiempo porque la investigación presentada en este informe, inició en el año 2018 y el período de tiempo que los investigadores establecieron para la revisión de bibliografía contempló los años anteriormente mencionados. Luego de seleccionar 32 estudios que permitieron obtener una lista de los impactos sociales más comunes de entre los reportados en la literatura analizada sobre el tema.

Para la selección, además, se verificaron los siguientes criterios: a) El estudio se centra en las consecuencias sociales de las actividades mineras, excluyendo la extracción de petróleo (pero incluyendo al carbón) y gas; b) Exclusión de estudios sobre temas más amplios, mismos que comúnmente no informan sobre indicadores directos que se relacionen con el impacto; c) Se procura presentar estudios con una amplia cobertura geográfica (se incluyeron estudios de todo el mundo); d) Se procuró analizar diversos artículos según ciertas categorías: e) 
ISSN: 2600-5859

Vol. 4., $\mathrm{N}^{\circ} 1.1$, p. 213-240, Febrero, 2021

Categoría de publicación, es decir "si fue artículo de revista, reportes u otros", Tipo de estudio, es decir si fue "Metodológico, Estudio de caso, Análisis estadístico, etc.", Qué producto se obtuvo "Oro, cobre, carbón, etc." y el nivel del análisis "escala Global, Nacional, escala Regional, escala Local”.

La selección de estas fuentes según los criterios empleados, se presentan en la tabla 1:

Tabla 1: Trabajos seleccionados de entre la literatura analizada y características principales.

\begin{tabular}{|c|c|c|c|c|c|c|c|}
\hline $\mathbf{N}^{\circ}$ & Autor & $\begin{array}{c}\text { Tipo de } \\
\text { publicación }\end{array}$ & $\begin{array}{l}\text { Tipo de } \\
\text { Filiación a }\end{array}$ & $\begin{array}{c}\text { Tipo } \\
\text { de } \\
\text { estudio }\end{array}$ & Región & Producto $^{b}$ & $\begin{array}{c}\text { Escala } \\
\text { del } \\
\text { estudio }\end{array}$ \\
\hline 1 & $\begin{array}{l}\text { The environmental } \\
\text { and socio-economic } \\
\text { impacts of mining } \\
\text { on local livelihoods } \\
\text { in Tanzania: A case } \\
\text { study of Geita } \\
\text { District. (Kitula, } \\
\text { 2006) }\end{array}$ & $\begin{array}{l}\text { Artículo de } \\
\text { revista }\end{array}$ & A. & $\begin{array}{l}\text { Caso } \\
\text { de } \\
\text { estudio }\end{array}$ & Tanzania & Oro & Local \\
\hline 2 & $\begin{array}{l}\text { Social dimensions } \\
\text { of mining: } \\
\text { Research, policy } \\
\text { and practice } \\
\text { challenges for the } \\
\text { minerals industry in } \\
\text { Australia. (Solomon } \\
\text { et al., 2008) }\end{array}$ & $\begin{array}{l}\text { Artículo de } \\
\text { revista }\end{array}$ & A. & $\begin{array}{l}\text { Revisió } \\
\mathrm{n} \text { de } \\
\text { literatur } \\
\mathrm{a}\end{array}$ & Australia & N/A & Nacional \\
\hline 3 & $\begin{array}{l}\text { Mining } \\
\text { developments and } \\
\text { social impacts on } \\
\text { communities: } \\
\text { Bowen Basin case } \\
\text { studies. (Petkova- } \\
\text { Timmer et al., } \\
\text { 2009) }\end{array}$ & $\begin{array}{l}\text { Artículo de } \\
\text { revista }\end{array}$ & A. & $\begin{array}{l}\text { Caso } \\
\text { de } \\
\text { estudio }\end{array}$ & Australia & N/A & Local \\
\hline 4 & $\begin{array}{l}\text { Demographic and } \\
\text { economic impact of } \\
\text { mining on remote } \\
\text { communities in } \\
\text { Australia. (Kotey \& } \\
\text { Rolfe, 2014) }\end{array}$ & $\begin{array}{l}\text { Artículo de } \\
\text { revista }\end{array}$ & A & $\begin{array}{l}\text { Estadíst } \\
\text { ico }\end{array}$ & Australia & N/A & Regional \\
\hline
\end{tabular}


Income Inequality across Australian

5 Regions during the Mining Boom:

2001-11. (Fleming

\& Measham, 2015)

Mining-induced displacement and

6 resettlement: a critical appraisal. (Owen \& Kemp, 2015)

Social impact

7 assessment of resource projects.

(Franks, 2012)

Mining and social development:

Refocusing

8 community investment using multi-criteria decision analysis. (Esteves, 2008)

Socio-economic wellbeing in Australian mining

towns: A

comparative analysis. (Tonts

et al., 2012)

Poverty in the Midst of Plenty:

Aboriginal People,

10 the 'Resource Curse' and Australia's Mining Boom. (Langton \&

Mazel, 2008)

Coal mining and the resource

11 community cycle: A longitudinal assessment of the
Artículo de revista

Artículo de revista

Reporte

Artículo de

revista

Artículo de revista

Artículo de revista

Artículo de revista
A. $\quad$ ico
Australia N/A
Regional

$\begin{array}{llll}\text { Caso } & & & \\ \text { estudio } & \text { N/A } & \text { N/A }\end{array}$

$\begin{array}{lll}\text { A. } & \begin{array}{l}\text { Metodo } \\ \text { lógico }\end{array} & \text { N/A }\end{array}$ N/A

$\begin{array}{lllll}\text { A. } & \text { Caso } & \text { Australia } & & \\ & \text { de } & - \text { Sud } & \text { N/A } & \text { Global } \\ & \text { estudio } & \text { África } & & \end{array}$
A. Estadíst ico
Australia N/A
Regional

Revisió

A. $\quad \begin{array}{llll}\text { literatur } & \text { Australia N/A Nacional }\end{array}$
A. de estudio
Australia Carbón
Local 
social impacts of the Coppabella coal mine. (Lockie et al., 2009)

Perspectives on community health 12 issues and the mining boom-bust cycle. (Shandro et al., 2011)

A multidisciplinary approach to understanding the 13 impacts of mines on traditional uses of water in Northern Mongolia.

(McIntyre et al., 2016)

Mining conflicts and Corporate

Social

14 Responsibility: Titanium mining in Kwale, Kenya.

(Abuya, 2016)

Mining,

Environment and

Community

Conflicts: A Study

of Company-

15

Community

Conflicts over Gold

Mining in the

Obuasi

Municipality of

Ghana. (Mensah \&

Okyere, 2014)

Indigenous peoples and non-ferrous

16 metals mining in the Philippines. (Holden, 2005)
Artículo de revista

Artículo de revista

Artículo de revista

Artículo de revista

Artículo de revista
Canadá
Carbón
Local estudio
A.
Caso Mongoli de
estudio
a
Oro
Nacional

A. $\begin{array}{llll}\text { Caso } & & \\ & \text { estudio } & & \text { Tenya }\end{array}$




\begin{tabular}{|c|c|c|c|c|c|c|c|}
\hline 17 & $\begin{array}{l}\text { Analysis of } \\
\text { Conflicts in the Use } \\
\text { of Space in Mining } \\
\text { Basin "Kolubara". } \\
\text { (Živković, 2012) }\end{array}$ & $\begin{array}{l}\text { Artículo de } \\
\text { revista }\end{array}$ & A. & $\begin{array}{l}\text { Revisió } \\
\mathrm{n} \text { de } \\
\text { literatur } \\
\text { a }\end{array}$ & Serbia & Lignito & Local \\
\hline 18 & $\begin{array}{l}\text { Lignite industry in } \\
\text { Greece within a } \\
\text { world context: } \\
\text { Mining, energy } \\
\text { supply and } \\
\text { environment. } \\
\text { (Kavouridis, 2008) }\end{array}$ & $\begin{array}{l}\text { Artículo de } \\
\text { revista }\end{array}$ & A. & $\begin{array}{l}\text { Revisió } \\
\mathrm{n} \text { de } \\
\text { literatur } \\
\mathrm{a}\end{array}$ & Grecia & Lignito & Nacional \\
\hline 19 & $\begin{array}{l}\text { Mineral resources } \\
\text { and localized } \\
\text { development: Q- } \\
\text { methodology for } \\
\text { rapid assessment of } \\
\text { socioeconomic } \\
\text { impacts in Rwanda. } \\
\text { (Weldegiorgis \& } \\
\text { Ali, 2016) }\end{array}$ & $\begin{array}{l}\text { Artículo de } \\
\text { revista }\end{array}$ & A. & $\begin{array}{l}\text { Estadíst } \\
\text { ico }\end{array}$ & Ruanda & Estaño & Local \\
\hline 20 & $\begin{array}{l}\text { The "battle of gold" } \\
\text { under the light of } \\
\text { green economics: a } \\
\text { case study from } \\
\text { Greece. (Damigos } \\
\text { \& Kaliampakos, } \\
\text { 2006) }\end{array}$ & $\begin{array}{l}\text { Artículo de } \\
\text { revista }\end{array}$ & A. & $\begin{array}{l}\text { Caso } \\
\text { de } \\
\text { estudio }\end{array}$ & Grecia & Oro & Local \\
\hline 21 & $\begin{array}{l}\text { Mining investment } \\
\text { and regional } \\
\text { development: A } \\
\text { scenario-based } \\
\text { assessment for } \\
\text { Northern Sweden. } \\
\text { (Ejdemo \& } \\
\text { Söderholm, 2011) }\end{array}$ & $\begin{array}{l}\text { Artículo de } \\
\text { revista }\end{array}$ & A. & $\begin{array}{l}\text { Estadíst } \\
\text { ico }\end{array}$ & Suecia & Oro, hierro & Local \\
\hline 22 & $\begin{array}{l}\text { Using input-output } \\
\text { analysis to estimate } \\
\text { the impact of a coal } \\
\text { industry expansion } \\
\text { on regional and } \\
\text { local economies. } \\
\text { (Ivanova \& Rolfe, } \\
\text { 2011) }\end{array}$ & $\begin{array}{l}\text { Artículo de } \\
\text { revista }\end{array}$ & A. & $\begin{array}{l}\text { Estadíst } \\
\text { ico }\end{array}$ & Australia & Carbón & Regional \\
\hline
\end{tabular}


ISSN: 2600-5859

\begin{tabular}{|c|c|c|c|c|c|c|c|}
\hline 23 & $\begin{array}{l}\text { Sustainability and } \\
\text { gold mining in the } \\
\text { developing world. } \\
\text { (Kumah, 2006) }\end{array}$ & $\begin{array}{l}\text { Artículo de } \\
\text { revista }\end{array}$ & A. & $\begin{array}{l}\text { Revisió } \\
\mathrm{n} \text { de } \\
\text { literatur } \\
\mathrm{a}\end{array}$ & Ghana & Oro & Global \\
\hline 24 & $\begin{array}{l}\text { Impacts of Surface } \\
\text { Gold Mining on } \\
\text { Land Use Systems } \\
\text { in Western Ghana. } \\
\text { (Schueler et al., } \\
2011 \text { ) }\end{array}$ & $\begin{array}{l}\text { Artículo de } \\
\text { revista }\end{array}$ & A. & $\begin{array}{l}\text { Caso } \\
\text { de } \\
\text { estudio }\end{array}$ & Ghana & Oro & Local \\
\hline 25 & $\begin{array}{l}\text { Social impacts of } \\
\text { mining: Changes } \\
\text { within the local } \\
\text { social landscape. } \\
\text { (Petrova \& } \\
\text { Marinova, 2013) }\end{array}$ & Otro & A. & $\begin{array}{l}\text { Caso } \\
\text { de } \\
\text { estudio }\end{array}$ & Australia & N/A & Local \\
\hline 26 & $\begin{array}{l}\text { Mining the Data: } \\
\text { Analyzing the } \\
\text { Economic } \\
\text { Implications of } \\
\text { Mining for } \\
\text { Nonmetropolitan } \\
\text { Regions. } \\
\text { (Freudenburg \& } \\
\text { Wilson, 2002) }\end{array}$ & $\begin{array}{l}\text { Artículo de } \\
\text { revista }\end{array}$ & A. & $\begin{array}{l}\text { Estadíst } \\
\text { ico }\end{array}$ & EE.UU. & N/A & Regional \\
\hline 27 & $\begin{array}{l}\text { Riding the } \\
\text { Resource Roller } \\
\text { Coaster: } \\
\text { Understanding } \\
\text { Socioeconomic } \\
\text { Differences } \\
\text { between Mining } \\
\text { Communities. } \\
\text { (Wilson, 2004) }\end{array}$ & $\begin{array}{l}\text { Artículo de } \\
\text { revista }\end{array}$ & A. & $\begin{array}{l}\text { Caso } \\
\text { de } \\
\text { estudio }\end{array}$ & EE.UU. & $\begin{array}{l}\text { Cobre, } \\
\text { plomo }\end{array}$ & Regional \\
\hline 28 & $\begin{array}{l}\text { Impacts and } \\
\text { development in } \\
\text { local economies } \\
\text { based on mining:: } \\
\text { the case of the } \\
\text { Chilean II region. } \\
\text { (Aroca, 2001) }\end{array}$ & $\begin{array}{l}\text { Artículo de } \\
\text { revista }\end{array}$ & A. & $\begin{array}{l}\text { Estadíst } \\
\text { ico }\end{array}$ & Chile & Cobre & Local \\
\hline
\end{tabular}




\begin{tabular}{|c|c|c|c|c|c|c|c|}
\hline 29 & $\begin{array}{l}\text { Sustainable } \\
\text { development } \\
\text { threats, inter-sector } \\
\text { conflicts and } \\
\text { environmental } \\
\text { policy requirements } \\
\text { in the arid, mining } \\
\text { rich, northern Chile } \\
\text { territory. (Oyarzún } \\
\text { \& Oyarzún, 2011) }\end{array}$ & $\begin{array}{l}\text { Artículo de } \\
\text { revista }\end{array}$ & A. & $\begin{array}{l}\text { Caso } \\
\text { de } \\
\text { estudio }\end{array}$ & Chile & Cobre & Nacional \\
\hline 30 & $\begin{array}{l}\text { Natural Resources } \\
\text { and Local } \\
\text { Communities: } \\
\text { Evidence from a } \\
\text { Peruvian Gold } \\
\text { Mine. (Aragón \& } \\
\text { Rud, 2013) }\end{array}$ & $\begin{array}{l}\text { Artículo de } \\
\text { revista }\end{array}$ & A. & $\begin{array}{l}\text { Caso } \\
\text { de } \\
\text { estudio }\end{array}$ & Perú & Oro & Local \\
\hline 31 & $\begin{array}{l}\text { Extractive } \\
\text { industries and local } \\
\text { development in the } \\
\text { Peruvian } \\
\text { Highlands. (Ticci \& } \\
\text { Escobal, 2015) }\end{array}$ & Reporte & A. & $\begin{array}{l}\text { Estadíst } \\
\text { ico }\end{array}$ & Perú & N/A & Regional \\
\hline 32 & $\begin{array}{l}\text { Mining and } \\
\text { campesino } \\
\text { engagement: an } \\
\text { opportunity for } \\
\text { integrated water } \\
\text { resources } \\
\text { management in } \\
\text { Ancash, Peru. } \\
\text { (Patrick \& } \\
\text { Bharadwaj, 2016) }\end{array}$ & $\begin{array}{l}\text { Artículo de } \\
\text { revista }\end{array}$ & A. & $\begin{array}{l}\text { Caso } \\
\text { de } \\
\text { estudio }\end{array}$ & Perú & N/A & Local \\
\hline
\end{tabular}

\section{Realizado por: Los autores}

La Tabla 1 muestra la lista de los estudios examinados, se observa que la mayoría son artículos publicados en revistas $(90,6 \%)$. Las áreas geográficas con más estudios son Australia $(31,2)$ y África $(21,88 \%)$. Resulta importante mencionar que mientras que en el $40,6 \%$ de los estudios revisados no se especifica el producto, el oro tiene la frecuencia más alta $(21,9 \%)$. La mayoría de las publicaciones se refieren a estudios de casos con un $46,9 \%$ de casos, y con el mismo porcentaje se presentan los estudios con escala del análisis local. 
Luego de analizar la información reportada en estos trabajos investigativos, se pudo determinar cuáles son las problemáticas sociales reportadas, dichos hallazgos sirvieron como insumos para la creación del cuestionario que permitirá analizar la precepción que los participantes del estudio tienen acerca de estos problemas. La lista de los problemas sociales reportados en los artículos y demás trabajos analizados se presenta en la tabla 2

\begin{tabular}{|c|c|c|c|c|c|}
\hline $\begin{array}{l}\text { Categoría } \\
\text { (según } \\
\text { impacto) }\end{array}$ & $\begin{array}{l}\text { Tipo de } \\
\text { impacto }\end{array}$ & Abreviación & $\begin{array}{c}\text { Local } \\
\text { (L) } \\
\text { Nacional } \\
\text { (N) }\end{array}$ & Descripción del impacto & $\begin{array}{l}\text { Fuente que } \\
\text { reporta } \\
\text { (según el } \\
\text { orden de la } \\
\text { tabla 1) }\end{array}$ \\
\hline \multirow[t]{8}{*}{$\begin{array}{l}\text { Economía, } \\
\text { ingresos y } \\
\text { seguridad }\end{array}$} & $\begin{array}{l}\text { Impactos } \\
\text { positivos }\end{array}$ & Ingresos & $\begin{array}{c}\mathrm{L} \\
\mathrm{N} \\
\mathrm{L}, \mathrm{N}\end{array}$ & $\begin{array}{l}\text { Contribución a los ingresos locales. } \\
\text { Mitigación de la pobreza. } \\
\text { Incremento de exportaciones y del } \\
\text { PIB. }\end{array}$ & $\begin{array}{l}1,7,8,9,19 \\
20,22,28,30\end{array}$ \\
\hline & & Negocios & $\mathrm{L}$ & $\begin{array}{c}\text { Oportunidades de negocios y } \\
\text { empleo en otros sectores (por la } \\
\text { revitalización de la economía y los } \\
\text { mercados). }\end{array}$ & $\begin{array}{c}1,3,20,22 \\
28\end{array}$ \\
\hline & $\begin{array}{l}\text { Impactos } \\
\text { negativos }\end{array}$ & Sobornos & $\mathrm{L}, \mathrm{N}$ & $\begin{array}{l}\text { Sobornos para obtener licencias y } \\
\text { permisos o para influir en una } \\
\text { decisión judicial y corrupción } \\
\text { (debido a una mala gestión de la } \\
\text { riqueza mineral). }\end{array}$ & 7 \\
\hline & & $\begin{array}{c}\text { Robos y } \\
\text { accidentes }\end{array}$ & $\mathrm{L}$ & $\begin{array}{l}\text { Accidentes y robos son comunes en } \\
\text { la comunidad minera. }\end{array}$ & 1 \\
\hline & & Desigualdad & $\begin{array}{l}\mathrm{L}, \mathrm{N} \\
\mathrm{L}\end{array}$ & $\begin{array}{l}\text { Desigualdad en los ingresos } \\
\text { generados. }\end{array}$ & $3,5,22$ \\
\hline & & & & $\begin{array}{c}\text { Bajo nivel de estímulo económico } \\
\text { de la minería debido a la } \\
\text { prevalencia de trabajadores no } \\
\text { residentes. }\end{array}$ & \\
\hline & & $\begin{array}{l}\text { Tensión } \\
\text { social }\end{array}$ & $\mathrm{L}$ & $\begin{array}{l}\text { Conflictos y tensiones sociales (por } \\
\text { la distribución desigual de } \\
\text { beneficios o el acceso limitado a los } \\
\text { recursos). }\end{array}$ & $\begin{array}{c}14,19,23,24 \\
29\end{array}$ \\
\hline & & & & $\begin{array}{l}\text { Conflictos entre empresas } \\
\text { constituidas legalmente y mineros }\end{array}$ & \\
\hline
\end{tabular}


ilegales; asesinatos por motivos

políticos de activistas contra la minería.

Pobreza

Empleo y Impactos

Educación positivos

Impactos

negativos

Trabajo

infantil

Malas

condiciones

laborales

Vulneración de derechos

laborales

Trabajos

temporales

Desempleo

Infraestructu

ra

suelo y

otros

aspectos

territoriale

Impactos

negativos
L Aumento de la pobreza debido a la pérdida de otros medios de subsistencia.

L Aumento de empleos (directos e indirectos en la comunidad local).

$3,7,11,19$, $21,22,30$

L Desarrollo de habilidades de los

28,31 empleados y posibilidad de capacitación y educación superior.

$\mathrm{L}$

Trabajo forzoso y obligatorio a infantes.

L Malas condiciones laborales, bajos

1,7 salarios.

L Falta de libertad para organizarse en $1,9,19$ sindicatos y no conformidad con los requisitos de los convenios de la Organización Internacional del

Trabajo.

L Creación de empleos en su mayoría temporales en comparación con los permanentes.

Baja estabilidad laboral.

L, N Aumento del índice de desempleo.

26,27

Mejoramiento de infraestructuras

$1,7,19,28$ como las de telecomunicaciones, red de carreteras, suministro de

L energía y agua.

Mejoramiento en al acceso a la salud y la educación.

Expropiaciones, desplazamiento y reasentamiento de la población, junto con el desempleo, falta de $1,3,7,14,15$, $17,18,19,23$, 24 tierra, falta de vivienda, pérdida de recursos comunes, empobrecimiento del nivel de vida. 
ISSN: 2600-5859

\begin{tabular}{|c|c|c|c|c|c|}
\hline & & $\begin{array}{l}\text { Acceso a la } \\
\quad \text { tierra }\end{array}$ & $\mathrm{L}$ & $\begin{array}{c}\text { Acceso limitado a la tierra, junto al } \\
\text { consiguiente impacto en los medios } \\
\text { de vida, inseguridad alimentaria y la } \\
\text { pérdida de áreas protegidas. }\end{array}$ & $\begin{array}{l}1,7,15,23 \\
24\end{array}$ \\
\hline \multirow[t]{3}{*}{$\begin{array}{c}\text { Demografí } \\
\text { a }\end{array}$} & $\begin{array}{l}\text { Impactos } \\
\text { positivos }\end{array}$ & $\begin{array}{l}\text { Crecimiento } \\
\text { de la } \\
\text { población }\end{array}$ & $\mathrm{L}$ & $\begin{array}{l}\text { Impactos positivos reportados por el } \\
\text { cambio demográfico, que genera } \\
\text { crecimiento de la población. }\end{array}$ & $4,31,25$ \\
\hline & $\begin{array}{l}\text { Impactos } \\
\text { negativos }\end{array}$ & $\begin{array}{l}\text { Desequilibri } \\
\text { o de género } \\
\text { y migración }\end{array}$ & $\mathrm{L}$ & $\begin{array}{c}\text { Gran afluencia migratoria y } \\
\text { desequilibrio de género en las } \\
\text { comunidades mineras, hechos que } \\
\text { hacen que aparezca el consiguiente } \\
\text { alcoholismo, drogas y prostitución, } \\
\text { propagación del VIH, violencia } \\
\text { doméstica, violencia sexual, cambio } \\
\text { en las normas sociales, cultura y } \\
\text { costumbres, migración, rotación de } \\
\text { la escuela secundaria. }\end{array}$ & $1,3,7,8,19$ \\
\hline & & Inflación & $\mathrm{L}$ & $\begin{array}{l}\text { Inflación, aumento de costos y } \\
\text { acceso al alojamiento para } \\
\text { trabajadores distintos de la minería. }\end{array}$ & $\begin{array}{c}3,7,8,11,19 \\
25,30\end{array}$ \\
\hline \multirow[t]{3}{*}{$\begin{array}{l}\text { Medio } \\
\text { Ambiente, } \\
\text { Salud y } \\
\text { Seguridad }\end{array}$} & $\begin{array}{l}\text { Impactos } \\
\text { negativos }\end{array}$ & $\begin{array}{l}\text { Competencia } \\
\text { por el uso } \\
\text { del agua }\end{array}$ & $\mathrm{L}$ & $\begin{array}{l}\text { Disminución del suministro de agua } \\
\text { y/o contaminación del agua, } \\
\text { competencia con otros usos (por } \\
\text { ejemplo, agricultura) y escasez de } \\
\text { agua. }\end{array}$ & $13,29,32$ \\
\hline & & $\begin{array}{l}\text { Impactos en } \\
\text { la salud }\end{array}$ & $\mathrm{L}$ & $\begin{array}{l}\text { Impactos negativos en la salud y en } \\
\text { la seguridad de la comunidad } \\
\text { minera (daños causados a las } \\
\text { viviendas por explosivos, lesiones } \\
\text { relacionadas con la mina durante el } \\
\text { auge de las actividades mineras). } \\
\text { Impactos sociales relacionados con } \\
\text { aspectos como aumento de los } \\
\text { embarazos, infecciones de } \\
\text { transmisión sexual, problemas de } \\
\text { salud mental como depresión y } \\
\text { ansiedad, estrés familiar, violencia } \\
\text { hacia las mujeres, etc. }\end{array}$ & $12,19,28$ \\
\hline & & $\begin{array}{l}\text { Impactos } \\
\text { ambientales } \\
\text { que afectan } \\
\text { la salud }\end{array}$ & $\mathrm{L}$ & $\begin{array}{l}\text { Impactos ambientales que afectan } \\
\text { las condiciones sociales y la salud. }\end{array}$ & $\begin{array}{c}1,7,11,12, \\
19,23,24,28\end{array}$ \\
\hline
\end{tabular}




\begin{tabular}{|c|c|c|c|c|c|}
\hline \multirow[t]{5}{*}{$\begin{array}{l}\text { Derechos } \\
\text { humanos }\end{array}$} & $\begin{array}{l}\text { Impactos } \\
\text { negativos }\end{array}$ & $\begin{array}{l}\text { Derechos } \\
\text { humanos }\end{array}$ & $\mathrm{L}$ & Abusos a los derechos humano. & $7,16,23$ \\
\hline & & $\begin{array}{l}\text { Recursos } \\
\text { culturales }\end{array}$ & $\mathrm{L}, \mathrm{N}$ & $\begin{array}{l}\text { Impactos negativos sobre los } \\
\text { recursos culturales. }\end{array}$ & 7 \\
\hline & & $\begin{array}{l}\text { Inclusión de } \\
\text { las partes } \\
\text { interesadas }\end{array}$ & $\mathrm{L}$ & $\begin{array}{c}\text { Falta de inclusión de las partes } \\
\text { interesadas y no participación de las } \\
\text { comunidades indígenas. }\end{array}$ & 6,7 \\
\hline & & $\begin{array}{c}\text { Discriminaci } \\
\text { ón }\end{array}$ & $\mathrm{L}$ & $\begin{array}{l}\text { Desigualdad de oportunidades y } \\
\text { discriminación por motivos de } \\
\text { género y marginación de grupos } \\
\text { vulnerables (discapacitados, } \\
\text { ancianos, minorías étnicas, } \\
\text { indígenas, jóvenes). }\end{array}$ & $6,7,14$ \\
\hline & & $\begin{array}{l}\text { Derechos } \\
\text { indígenas }\end{array}$ & $\mathrm{L}$ & $\begin{array}{l}\text { Irrespeto a los derechos de los } \\
\text { pueblos indígenas. }\end{array}$ & 16 \\
\hline
\end{tabular}

Tabla 2: Lista de impactos sociales reportados en la bibliografía analizada Realizado por: los autores

La determinación de los indicadores reportados como problemas sociales, indicados en la tabla 2 permitió generar la encuesta que fue aplicada a los participantes del estudio con la finalidad de medir su percepción con respecto a dichos aspectos. Tanto el desarrollo de la prueba de campo efectuada como los resultados que arrojó el análisis de los datos obtenidos se presentan en las siguientes secciones.

\section{Metodologia.}

Según Sampieri et al. (2014), la elección apropiada del diseño de un estudio, facilitará la adecuada adaptación y conexión de todas las etapas de un proceso de investigación como son el análisis y el planteamiento del problema, el estudio del estado de la cuestión y/o marco teórico, así como también ayuda en la conexión lógica que debe existir entre las hipótesis y las fases posteriores del estudio, mismas que se presentan con un carácter más operativo que teórico. Cabe señalar que este estudio presenta un enfoque mixto, ya que emplea más de un método para su ejecución, así pues, éste fue un estudio transversal y se desarrolló en un escenario que involucró la participación de 72 personas que habitan en el cantón Camilo Ponce Enríquez cuyos ingresos dependen directa o indirectamente de las actividades mineras en esta zona de nuestro país, el interés de los investigadores se centró en conocer la percepción de estas personas con respecto a los problemas sociales que causa la minería a nivel global que fueron reportados en la bibliografía analizada, la muestra empleada fue obtenida empleando muestro no probabilístico, estratificado intencional y discrecional, 
siendo que los investigadores tuvieron que entrevistar a los participantes del estudio mediante la encuesta que se generó a partir de los datos reportados en la revisión bibliográfica.

Los investigadores procuraron conformar la muestra de tal forma que fuese representativa de la población tanto en el número de personas que la integraron como en las características cualitativas que distinguen a los ciudadanos del cantón Camilo Ponce Enríquez que se dedican a las actividades mineras. El proceso mediante el cual se obtuvo la muestra se detalla a continuación:

Según Jorge Arias, representante de la compañía Minervilla y de la Asociación de Mineros 12 de Octubre, que operan en el cantón Camilo Ponce Enríquez, en la provincia de Azuay, en el cantón existen 5000 personas cuyos ingresos dependen directa o indirectamente de las actividades mineras (Diario el Universo, 2020), en base a esta cantidad se procedió a emplear la siguiente fórmula para conocer el número de personas que debían conformar la muestra del estudio.

$$
n=\frac{N * Z_{a}^{2} * p * q}{d^{2} *(N-1)+Z_{a}^{2} * p * q}
$$

Fórmula para calcular la muestra conociendo el número de elementos de la población

En donde: $\mathrm{N}$ = tamaño de la población; $\mathrm{Z}=$ nivel de confianza; $\mathrm{p}=$ probabilidad de éxito $\mathrm{o}$ proporción esperada; $\mathrm{q}$ = probabilidad de fracaso; $\mathrm{d}$ = precisión (Error máximo admisible en términos de proporción). Para el caso puntual de este estudio, se emplea una fiabilidad del 95\% $(\mathrm{Z}=1,96)$, con una probabilidad de éxito de $\mathrm{p}=5 \%$ y con los datos indicados anteriormente donde $\mathrm{N}=5000$, se obtiene que:

\begin{tabular}{lcc} 
& \multicolumn{2}{c}{ Tabla 3: Valores para cálculo de la muestra } \\
\cline { 2 - 3 } & Parámetro & Valor \\
& $\mathrm{N}$ & 5000 \\
& $\mathrm{Z}$ & 1,960 \\
& $\mathrm{p}$ & $5 \%$ \\
& $\mathrm{q}$ & $95 \%$ \\
& $\mathrm{~d}$ & $5,00 \%$ \\
& &
\end{tabular}

Aplicando la formula se obtiene que $\mathrm{n}=71.95$, entonces se trabaja con una muestra compuesta por 72 sujetos. 
El presente trabajo de investigación utilizó el método bibliográfico mediante el cual se revisaron artículos académicos sobre el tema de interés que fueron publicados en bases de datos científicas especializadas, dicha búsqueda pretendía la consecución de información pertinente, válida y relevante sobre los problemas sociales reportados a nivel internacional. Esta actividad derivó en el diseño de la encuesta que permitiría analizar la percepción de los participantes del estudio acerca de los problemas comunes encontrados

Antes de ejecutar el estudio, es decir en la fase de diseño del experimento de la investigación, se definieron de forma clara y concisa tanto la organización operativa del trabajo como la metodología empleada y los ejes específicos orientan la investigación. De la misma manera, se analizó la aproximación al objeto de estudio mediante la revisión de bibliografía que permitió la construcción del marco teórico y del estado del arte del tema de estudio, seguidamente se procedió en base a dicha aproximación teórica a delimitar el trabajo para concretar cada uno de los elementos y las variables que se estudiarían y analizaría en las etapas posteriores.

La recopilación y posterior análisis de información publicada en artículos sobre el tema, permitió definir problemas sociales latentes más comunes reportados a nivel global que causan las actividades mineras, el conocimiento concreto de estas problemáticas detectadas permitió generar la encuesta para recabar información útil que permita conocer la percepción de las personas del cantón Camilo Ponce Enríquez (que de una u otra forma están ligados a las actividades mineras), con respecto a las ya mencionadas problemáticas a partir de las respuestas proporcionadas por éstos ciudadanos.

Luego de la fase de indagación, se tabularon y analizaron las respuestas a las preguntas de la encuesta en busca de información relevante para los propósitos del estudio. Los resultados del trabajo reportado se presentan en la siguiente sección.

\section{Resultados.}

La investigación pretende dar una visión sobre la percepción de un grupo de personas $(\mathrm{n}=72)$ que realizan actividades mineras en el cantón Camilo Ponce Enríquez, en la provincia de Azuay, Ecuador, los investigadores desde la fase de análisis de la bibliografía revisada, han seleccionado las siguientes dimensiones:

- Economía, ingresos y seguridad

- Empleo y Educación

- Uso del suelo y otros aspectos territoriales

- Demografía

- Medio Ambiente, Salud y Seguridad

- Derechos humanos 
Para la obtención de los resultados del estudio, se elaboró una encuesta cerrada, en la que cada participante debía seleccionar 1 opción para cada pregunta dentro de los indicadores en que se constituyó la encuesta del trabajo. El instrumento diseñado se aplicó en campo a cada uno de los 72 sujetos que integraron la muestra, entre el 5 de septiembre de 2020 y el 25 septiembre de 2020. Se levantó la información a partir de los datos recopilados en las 72 encuestas con los criterios antes mencionados. A partir del tipo de muestreo empleado (muestro no probabilístico, estratificado intencional y discrecional), se usó la encuesta y se obtuvieron los resultados correspondientes a los aspectos reportados como problemas (impactos negativos) que se presentan a continuación:

Tabla 3: Economía, ingresos y seguridad

\begin{tabular}{ll}
\hline Temáticas según su importancia & Porcentaje en función de la frecuencia \\
\hline 1 Sobornos & Tensión Social \\
2 Robos y accidentes & $34,72 \%$ \\
3 Desigualdad & Sobornos \\
4 Tensión Social & $19,44 \%$ \\
5 Pobreza & Pobreza \\
& $19,44 \%$ \\
& Desigualdad \\
& $16,70 \%$ \\
& Robos y Accidentes \\
\hline
\end{tabular}

Como se aprecia en los resultados de la pregunta correspondiente al indicador 1, la tensión social generada se percibe como el problema principal en el ámbito de las actividades mineras en el cantón ya que resulta incómodo para las personas encuestadas la manera desigual en que se reparten los beneficios que genera la actividad minera, además existen fuertes conflictos entre las empresas constituidas legalmente y los mineros ilegales de la zona, situación que genera enfrentamientos e incluso muertes por este tipo de problema. En segundo lugar se reporta que los accidentes en las minas y los sobornos realizados para la obtención de licencias o para influir en decisiones judiciales aunados a temas de corrupción es el segundo problema en importancia reportado por los encuestados, con el mismo porcentaje se reporta al aumento de la pobreza ya que con la actividad minera se pierden, según la percepción de los encuestados otros medios de subsistencia siendo la actividad minera la fuente principal de ingresos y sustento de la zona. Finalmente, pero en que dejen de ser preocupantes, se reportan la desigualdad percibida en los ingresos generados 
(reportado también como el indicador más preocupante) y los robos y accidentes que son hechos comunes dentro de la comunidad minera.

Tabla 4: Empleo y Educación.

\begin{tabular}{ll}
\hline Actividades según su importancia & Porcentaje en función de la frecuencia \\
\hline 1.- Trabajo infantil & Trabajos temporales \\
& $30,6 \%$ \\
2.- Malas condiciones laborales & Desempleo \\
3.- Vulneración de derechos laborales & $30,6 \%$ \\
4.- Trabajos temporales & Malas condiciones laborales \\
5.- Desempleo & $19,4 \%$ \\
& Vulneración de derechos laborales \\
& $15,3 \%$ \\
& Trabajo infantil \\
\hline
\end{tabular}

$\mathrm{Al}$ analizar los resultados de la pregunta correspondiente al indicador 2, se aprecia que con respecto al empleo y educación las personas encuestadas perciben como el mayor problema a la modalidad de trabajo que mayoritariamente es temporal, generando inseguridad y una baja estabilidad laboral, este aspecto se liga con el segundo problema reportado en las respuesta de la encuesta, el desempleo, mismo que se presenta con el mismo porcentaje que el problema más preocupante en este indicador, a estos le siguen las malas condiciones de trabajo reportándose principalmente un descontento por los bajos salarios que perciben los obreros que se desempeñan en el ámbito minero del sector. Como consecuencia de los aspectos mencionados, se reporta también malestar por la imposibilidad que tienen las personas involucradas para organizarse en sindicatos de obreros. De igual forma, en un porcentaje pequeño se reporta como preocupante a la existencia de casos en que los trabajos mineros son desempeñados por infantes. Lo cual constituye una violación a sus derechos.

Tabla 5: Uso del suelo y otros aspectos territoriales.

Ítems según su importancia $\quad$ Porcentaje en función de la frecuencia

\begin{tabular}{ll}
\hline 1.- Expropiación / desplazamiento & Acceso a la tierra \\
& $65,3 \%$
\end{tabular}


En cuanto a los resultados obtenidos sobre el indicador uso del suelo, se observa que la literatura revisada solo reportó dos problemas mismos que son el acceso limitado a la tierra, que se reporta en la encuesta del estudio con un porcentaje mayor, lo que hace notar la preocupación de las personas encuestadas junto al impacto que conlleva esta situación en los medios de vida de dichas personas y sus familias, tales como la inseguridad alimentaria y/o la pérdida de áreas protegidas, el segundo problema reportado es la expropiación de tierras y el consecuente desplazamiento que este hecho acarrea para las personas de la zona para quienes es evidente la falta de tierras y de vivienda que entre otras cosas, causa el empobrecimiento y deterioro de la calidad de vida.

Tabla 6: Demografía

Ítems según su importancia $\quad$ Porcentaje en función de la frecuencia

1.- Desequilibrio de género y migración Vivienda

2.- Vivienda

$$
84,7 \%
$$

Desequilibrio de género y migración $15,3 \%$

En cuanto a los temas demográficos la precepción reportada por los informantes del estudio evidencia que la inflación es el problema más latente ya que según se reflejan en los datos recabados es evidente que en la zona minera analizada existe un aumento en los costos y en lo referente al alojamiento y la renta de viviendas para las personas, es así que este indicador se presenta con un porcentaje mayor que el otro factor reportado en la literatura revisada para este ítem, como es el Desequilibrio de género y migración, que si bien es cierto se reporta en mucha menor medida parece inquietar a los encuestados sobre todo en temas de alcoholismo, drogas, prostitución, aumento en los casos de enfermedades de transmisión sexual, violencia (intrafamiliar y/o sexual), alienación, migración, entre otras.

Tabla 7: Medio Ambiente, Salud y Seguridad

Metas según su importancia $\quad$ Porcentaje en función de la frecuencia

1.- Competencia por el uso del agua

Competencia por el uso del agua

$44,4 \%$ 
2.- Impactos en la salud

3.- Impactos ambientales que afectan la salud
Impactos en la salud

$30,6 \%$

Impactos ambientales que afectan la salud $25 \%$

Con respecto a los problemas reportados para el indicador Medio Ambiente, Salud y Seguridad se nota una preocupación mayor en lo que se refiere al acceso al agua y a la contaminación del líquido vital y que las actividades mineras compiten directamente con otras actividades importantes como la agricultura por el acceso al agua, recurso que se percibe como escaso. Se reporta también como preocupante el aspecto de los impactos que tiene la actividad minera en la salud de las personas, principalmente causados por accidentes en las minas por el uso de explosivos, así mismo resulta preocupante el aumento de los embarazos, enfermedades de transmisión sexual, problemas mentales, estrés familiar, violencia de género, etc. En menor medida se reporta también la preocupación por los impactos ambientales que produce la actividad minera y que tienen repercusiones en la salud de las personas tales como la contaminación por mercurio, y la afección que este hecho causa en la flora y fauna del entorno próximo a la explotación minera y, los efectos negativos que tiene en la salud de las personas que habitan en las poblaciones próximas a las minas (aunque, en ocasiones estos efectos pueden extenderse más allá de dichos poblados).

Tabla 8: Derechos humanos

\begin{tabular}{ll}
\hline Metas según su importancia & Porcentaje en función de la frecuencia \\
\hline 1.- Derechos humanos & Inclusión de las partes interesadas \\
& $44,4 \%$ \\
2.- Recursos culturales & Derechos humanos \\
3.- Inclusión de las partes interesadas & $30,6 \%$ \\
& Recursos culturales \\
& $25 \%$ \\
\hline
\end{tabular}

La percepción de los encuestados con respecto al sexto indicador, Derechos humanos evidencian que el mayor problema es la inclusión de las partes interesadas en las actividades mineras, hecho que se evidencia por ejemplo en la no inclusión de personas de comunidades indígenas de la zona, la mayoría de los trabajadores son foráneos al cantón. En segundo lugar, se reporta como una preocupación a la presencia de hechos considerados como abusos a los 
derechos humanos tales como los ligados a desalojos forzosos de los pobladores de la zona y al control territorial de las empresas mineras, apropiación de tierras, entre otros, finalmente en orden de importancia se reporta la existencia de impactos negativos sobre los recursos culturales de la zona debido a la migración que produce la actividad minera, hecho que hace que personas de otras culturas y costumbres convivan con la gente de la zona y que se modifiquen o alteren las costumbres y por tanto la cultura de estos pueblos.

\section{Conclusiones.}

- El modelo económico de la zona debe cambiar su enfoque y orientarse principalmente al bienestar de los ciudadanos de la zona, de esta manera es importante la concepción de que la estructura social y la estructura económico-productiva no puede entenderse con un fin en sí misma, sino que es un medio por el cual se mejoren las condiciones de vida de las personas que habitan la zona, beneficiando y brindando bienestar a la comunidad del cantón. En ese sentido, las interacciones económico-productivas que se derivan a de la actividad minera en la zona, deben promover una economía de "retorno circular" y con enfoque solidario.

- Las culturas indígenas que se hallan apostadas en estos territorios y la herencia ideológica y cultural que se creó durante cientos de años con un complejo proceso de mestizaje, así como la migración campo-ciudad y desde el extranjero que generan las actividades mineras, entre otros aspectos hacen que esta región del país sea una zona muy diversa. Con esto en mente, es importante el desarrollo de iniciativas y programas que centren su acción a la sensibilización, la tolerancia y la educación, trabajar en estos ejes con los grupos vulnerables influirá positivamente en este componente, es así que los gobiernos seccionales del cantón y de la zona deben estar conscientes y decididos a realizar el esfuerzo que amerita la protección de las personas en situación de movilidad, a las que son víctimas de violencia de género y/o a las personas discriminadas por aspectos raciales, culturales, religiosos, etc., entre otros aspectos.

- Otro aspecto importante a considerar para conseguir desarrollo adecuado y sustentable en la zona es la organización adecuada de los asentamientos humanos, la movilidad, la provisión de energía y de telecomunicaciones, por ello es necesario que el GAD municipal, dentro del marco de su competencias garantice una distribución de asentamientos humanos de acuerdo a las formas que se establecen por parte del Código Orgánico de Organización Territorial (COOTAD), considerando a la vez, los riesgos potenciales (ambientales y de salud) que se generarían si no se diferencian las zonas cuyos suelo debe ser de utilizado para el desarrollo de la actividad minera, diferenciándolos de los suelos que se utilizarán como espacios verdes, para producción agrícola, para espacios recreativos, y de las zonas aptas para habitar.

- Es importante la creación y mantenimiento de infraestructura que garantice la provisión de servicios básicos, creación que vaya de la mano con políticas que consideren los procesos migratorios que la actividad minera genera en la zona, dicha provisión de servicios hará entre otras cosas que sea posible una comunicación de calidad, aspectos importantes para el bienestar de la comunidad que deben ser priorizados. 
- El trabajo por hacer es grande y tiene varias aristas que deben ser atendidas, la meta a conseguir sería que el cantón tenga como misión el convertirse en un territorio que cuente con bases sólidas que permitan el desarrollo de la actividad minera en mejores condiciones, escenario al que se llegará mediante cambios progresivos en la dinámica social. económica y productiva, mediante el dialogo y la concreción de acuerdos entre todas las partes involucradas, para conseguir hacer del cantón un territorio que permita el desarrollo de las actividades mineras en un entorno social inclusivo, sostenible, intercultural y próspero. En general los investigadores consideran que la evaluación de los impactos negativos se presenta como un desafío importante en la evaluación de la sostenibilidad de esta importante actividad económica del cantón.

\section{Referencias bibliográficas.}

Aldana, S., Vereda, F., Hidalgo-Alvarez, R., \& de Vicente, J. (2016). Facile synthesis of magnetic agarose microfibers by directed selfassembly. Polymer, 93, 61-64.

Bhat, S., Tripathi, A., \& Kumar, A. (2010). Supermacroprous chitosan-agarose-gelatin cryogels. in vitro characterization and in vivo assesment for cartilage tissue engineering. Journal of the Royal Society Interface, 1-15.

Bossis, G., Marins, J., Kuzhir, P., Volkova, O., \& Zubarev, A. (2015). Functionalized microfibers for field-responsive materials and biological applications. Journal of Intelligent Material Systems and Structures, 1-9.

Cortés, J., Puig, J., Morales , J., \& Mendizábal, E. (2011). Hidrogeles nanoestructurados termosensibles sintetizados mediante polimerización en microemulsión inversa. Revista Mexicana de Ingeniería Química., 10(3), 513-520.

Dias, A., Hussain, A., Marcos, A., \& Roque, A. (2011). A biotechnological perspective on the application of iron oxide magnetic colloids modified with polysaccharides. Biotechnology Advances 29, 29, 142-155.

Estrada Guerrero, R., Lemus Torres, D., Mendoza Anaya, D., \& Rodriguez Lugo, V. (2010). Hidrogeles poliméricos potencialmente aplicables en Agricultura. Revista Iberoamericana de Polímeros, 12(2), 76-87.

García-Cerda, L., Rodríguez-Fernández, O., Betancourt-Galindo, R., Saldívar-Guerrero, R., \& Torres-Torres, M. (2003). Síntesis y propiedades de ferrofluidos de magnetita. Superficies y Vacio., 16(1), 28-31.

Ilg, P. (2013). Stimuli-responsive hydrogels cross-linked by magnetic nanoparticles. Soft Matter, 9, 3465-3468. 
Lewitus, D., Branch, J., Smith, K., Callegari, G., Kohn, J., \& Neimark, A. (2011). Biohybrid carbon nanotube/agarose fibers for neural tissue engineering. Advanced Functional Materials, 21, 2624-2632.

Lin, Y.-S., Huang, K.-S., Yang, C.-H., Wang, C.-Y., Yang, Y.-S., Hsu, H.-C., . . Tsai, C.W. (2012). Microfluidic synthesis of microfibers for magnetic-responsive controlled drug release and cell culture. PLOS ONE, 7(3), 1-8.

Ruiz Estrada, G. (2004). Desarrollo de un Sistema de liberación de fármacos basado en nanopartículas magnéticas recubiertas con Polietilénglicol para el tratamiento de diferentes enfermedades. Madrid: Universidad Autónoma de Madrid. Departamento de Física Aplicada.

Song, J., King, S., Yoon, S., Cho, D., \& Jeong, Y. (2014). Enhanced spinnability of narbon nanotube fibers by surfactant addition. Fiberes and Polymers, 15(4), 762-766.

Tartaj, P., Morales, M., González-Carreño, T., Veintemillas-Verdaguer, S., \& Serna, C. (2005). Advances in magnetic nanoparticles for biotechnology applications. Journal of Magnetism and Magnetic Materials, 290, 28-34.

Wulff-Pérez , M., Martín-Rodriguez, A., Gálvez-Ruiz, M., \& de Vicente, J. ( 2013 ). The effect of polymer surfactant on the rheological properties of nanoemulsions. Colloid and Polymer Science, 291, 709-716.

Zamora Mora, V., Soares, P., Echeverria, C., Hernández , R., \& Mijangos, C. (2015). Composite chitosan/Agarose ferrogels for potential applications in magnetic hyperethermia. Gels., 1, 69-80.

Abuya, W. O. (2016). Mining conflicts and Corporate Social Responsibility: Titanium mining in Kwale, Kenya. The Extractive Industries and Society, 3(2), 485-493. https://doi.org/10.1016/j.exis.2015.12.008

Aragón, F. M., \& Rud, J. P. (2013). Natural Resources and Local Communities: Evidence from a Peruvian Gold Mine. American Economic Journal: Economic Policy, 5(2), 1 25.

Aroca, P. (2001). Impacts and development in local economies based on mining: The case of the Chilean II region. Resources Policy, 27(2), 119-134. https://doi.org/10.1016/S0301-4207(01)00013-7

Damigos, D., \& Kaliampakos, D. (2006). The "battle of gold" under the light of green economics: A case study from Greece. Environmental Geology, 50(2), 202-218. https://doi.org/10.1007/s00254-006-0201-9 
Dewulf, J., Blengini, G. A., Pennington, D., Nuss, P., \& Nassar, N. T. (2016). Criticality on the international scene: Quo vadis? Resources Policy, 50, 169-176. https://doi.org/10.1016/j.resourpol.2016.09.008

Diario el Universo. (2020, septiembre 21). Trabajadores mineros de Camilo Ponce Enríquez reclaman por fallo judicial que dispone devolución de concesionarias incautadas. El Universo.

https://www.eluniverso.com/noticias/2020/09/21/nota/7986160/trabajadoresmineros-camilo-ponce-enriquez-reclaman-fallo-judicial

Ejdemo, T., \& Söderholm, P. (2011). Mining investment and regional development: A scenario-based assessment for Northern Sweden. Resources Policy, 36(1), 14-21. https://doi.org/10.1016/j.resourpol.2010.08.008

Esteves, A. M. (2008). Mining and social development: Refocusing community investment using multi-criteria decision analysis. Resources Policy, 33(1), 39-47. https://doi.org/10.1016/j.resourpol.2008.01.002

Fleming, D. A., \& Measham, T. G. (2015). Income Inequality across Australian Regions during the Mining Boom: 2001-11. Australian Geographer, 46(2), 203-216. https://doi.org/10.1080/00049182.2015.1020596

Franks, D. (2012). Social impact assessment of resource projects. International Mining for Development Centre, 16.

Freudenburg, W. R., \& Wilson, L. J. (2002). Mining the Data: Analyzing the Economic Implications of Mining for Nonmetropolitan Regions. Sociological Inquiry, 72(4), 549-575. https://doi.org/10.1111/1475-682X.00034

Holden, W. (2005). Indigenous peoples and non-ferrous metals mining in the Philippines:

The Pacific Review: Vol 18, No 3. https://www.tandfonline.com/doi/abs/10.1080/09512740500189199

Ivanova, D. G., \& Rolfe, D. J. (2011). Using input-output analysis to estimate the impact of a coal industry expansion on regional and local economies. Impact Assessment and Project Appraisal, 29(4), 277-288. https://doi.org/10.3152/146155111X12959673795840

Kavouridis, K. (2008). Lignite industry in Greece within a world context: Mining, energy supply and environment. Energy Policy, 36(4), 1257-1272. https://doi.org/10.1016/j.enpol.2007.11.017 
Kitula, A. G. N. (2006). The environmental and socio-economic impacts of mining on local livelihoods in Tanzania: A case study of Geita District. Journal of Cleaner Production, 14(3), 405-414. https://doi.org/10.1016/j.jclepro.2004.01.012

Kotey, B., \& Rolfe, J. (2014). Demographic and economic impact of mining on remote communities in Australia. Resources Policy, 42, 65-72. https://doi.org/10.1016/j.resourpol.2014.10.005

Kumah, A. (2006). Sustainability and gold mining in the developing world. Journal of Cleaner Production, 14(3-4), 315-323. https://doi.org/10.1016/j.jclepro.2004.08.007

Langton, M., \& Mazel, O. (2008). Poverty in the Midst of Plenty: Aboriginal People, the 'Resource Curse' and Australia's Mining Boom. Journal of Energy \& Natural Resources Law, 26(1), 31-65. https://doi.org/10.1080/02646811.2008.11435177

Lockie, S., Franettovich, M., Petkova-Timmer, V., Rolfe, J., \& Ivanova, G. (2009). Coal mining and the resource community cycle: A longitudinal assessment of the social impacts of the Coppabella coal mine. Environmental Impact Assessment Review, 29(5), 330-339. https://doi.org/10.1016/j.eiar.2009.01.008

McIntyre, N., Bulovic, N., Cane, I., \& McKenna, P. (2016). A multi-disciplinary approach to understanding the impacts of mines on traditional uses of water in Northern Mongolia. Science of The Total Environment, 557-558, 404-414. https://doi.org/10.1016/j.scitotenv.2016.03.092

Mensah, S. O., \& Okyere, S. A. (2014). Mining, Environment and Community Conflicts: A Study of Company-Community Conflicts over Gold Mining in the Obuasi Municipality of Ghana. Journal of Sustainable Development Studies, 5(1), Article 1. https://infinitypress.info/index.php/jsds/article/view/537

Owen, J. R., \& Kemp, D. (2015). Mining-induced displacement and resettlement: A critical appraisal. Journal of Cleaner Production, 87, 478-488. https://doi.org/10.1016/j.jclepro.2014.09.087

Oyarzún, J., \& Oyarzún, R. (2011). Sustainable development threats, inter-sector conflicts and environmental policy requirements in the arid, mining rich, northern Chile territory. Sustainable Development, 19(4), 263-274. https://doi.org/10.1002/sd.441

Patrick, R., \& Bharadwaj, L. (2016). Mining and campesino engagement: An opportunity for integrated water resources management in Ancash, Peru. Water International, 41(3), 468-482. https://doi.org/10.1080/02508060.2016.1160311 
Petkova-Timmer, V., Lockie, S., Rolfe, J., \& Ivanova, G. (2009). Mining Developments and Social Impacts on Communities: Bowen Basin Case Studies: Rural Society: Vol 19, No 3. Rural Society, 19(3), 211-228.

Petrova, S., \& Marinova, D. (2013). Social impacts of mining: Changes within the local social landscape. Rural Society, 22(2), 153-165. https://doi.org/10.5172/rsj.2013.22.2.153

Ríos, R. V. (2018). Minería en América Latina y el Caribe, un enfoque socioambiental. Revista UDCA Actualidad \& Divulgación Científica, 21(2), 617-631.

Rosa, W. (Ed.). (2017). Transforming Our World: The 2030 Agenda for Sustainable Development. En A New Era in Global Health. Springer Publishing Company. https://doi.org/10.1891/9780826190123.ap02

Saade Hazin, M. (2013). Desarrollo minero y conflictos socioambientales: Los casos de Colombia, México y el Perú. https://repositorio.cepal.org//handle/11362/5369

Sampieri, R., Fernández, C., \& Baptista, P. (2014). Metodología de la Investigación (Sexta). McGraw-Hill Interamericana.

Schueler, V., Kuemmerle, T., \& Schröder, H. (2011). Impacts of Surface Gold Mining on Land Use Systems in Western Ghana. AMBIO, 40(5), 528-539. https://doi.org/10.1007/s13280-011-0141-9

Shandro, J. A., Veiga, M. M., Shoveller, J., Scoble, M., \& Koehoorn, M. (2011). Perspectives on community health issues and the mining boom-bust cycle. Resources Policy, 36(2), 178-186. https://doi.org/10.1016/j.resourpol.2011.01.004

Solomon, F., Katz, E., \& Lovel, R. (2008). Social dimensions of mining: Research, policy and practice challenges for the minerals industry in Australia. Resources Policy, 33(3), 142-149. https://doi.org/10.1016/j.resourpol.2008.01.005

Ticci, E., \& Escobal, J. (2015). Extractive industries and local development in the Peruvian Highlands. Environment and Development Economics, 20(1), 101-126. https://doi.org/10.1017/S1355770X13000685

Toapanta, A. R. R. (2017). Política minera y sostenibilidad ambiental en Ecuador. FIGEMPA: Investigación y Desarrollo, 1(2), 41-52. https://doi.org/10.29166/revfig.v1i2.68

Tonts, M., Plummer, P., \& Lawrie, M. (2012). Socio-economic wellbeing in Australian mining towns: A comparative analysis. Journal of Rural Studies, 28(3), 288-301. https://doi.org/10.1016/j.jrurstud.2011.10.006 
Twerefou, D. K. (2009). Mineral Exploitation, Environmental Sustainability and Sustainable Development in EAC, SADC, and ECOWAS Regions. African Trade Policy Centre, Economic Commission for Africa. https://www.researchgate.net/publication/260929736_Mineral_exploitation_environ mental_sustainability_and_sustainable_development_in_EAC_SADC_and_ECOW AS_Regions

Weldegiorgis, F. S., \& Ali, S. H. (2016). Mineral resources and localised development: Qmethodology for rapid assessment of socioeconomic impacts in Rwanda. Resources Policy, 49, 1-11. https://doi.org/10.1016/j.resourpol.2016.03.006

Wilson, L. J. (2004). Riding the Resource Roller Coaster: Understanding Socioeconomic Differences between Mining Communities*. Rural Sociology, 69(2), 261-281. https://doi.org/10.1526/003601104323087606

Živković, M. (2012). Analysis of Conflicts in the Use of Space in Mining Basin "Kolubara". Зборник Радова Географског Института “Јован Цвијић” САНУ, 62(3), 123-136. 


\section{PARA CITAR EL ARTÍCULO INDEXADO.}

Matute Saquicela, G. E., Covri Rivera, D., \& Castillo Ortega, Y. (2021). Incidencia de la actividad minera en el desarrollo local sostenible: el caso del Cantón Camilo Ponce Enríquez. ConcienciaDigital, 4(1.1), 213-240. https://doi.org/10.33262/concienciadigital.v4i1.1.1555

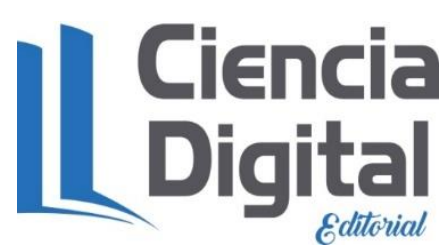

El artículo que se publica es de exclusiva responsabilidad de los autores y no necesariamente reflejan el pensamiento de la Revista Ciencia Digital.

El artículo queda en propiedad de la revista y, por tanto, su publicación parcial y/o total en otro medio tiene que ser autorizado por el director de la Revista Ciencia Digital.
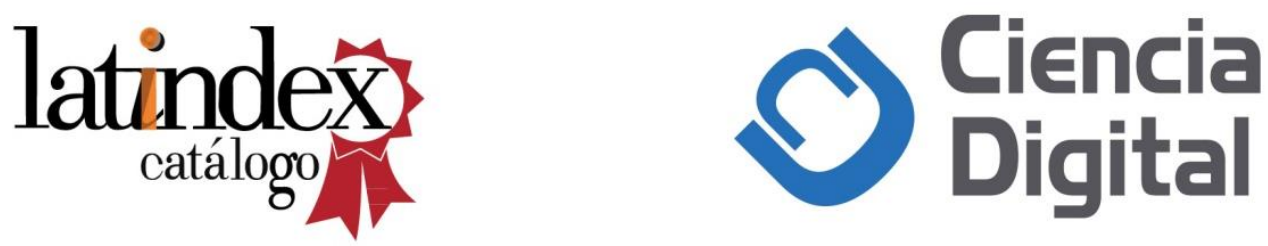